\title{
A WORD-LENGTH-DEPENDENT CONFIDENCE MEASURE FOR LARGE VOCABULARY CHINESE KEYWORD SPOTTING
}

\author{
Bo-Ren Bai ${ }^{l}$, Hsin-Min Wang ${ }^{2}$ and Lin-Shan Lee ${ }^{1,2}$ \\ ${ }^{1}$ Dept. of Electrical Engineering, National Taiwan University \\ ${ }^{2}$ Institute of Information Science, Academia Sinica \\ Taipei, Taiwan, R.O.C. \\ E-mail: white@speech.ee.ntu.edu.tw
}

\begin{abstract}
In this paper, a word-length-dependent confidence measure for large vocabulary Chinese keyword spotting is proposed to deal with the problem caused by the significantly difference in keyword length, i.e. false alarms are likely to occur for shorter keywords, while false rejections are more likely to occur for longer keywords. The proposed confidence measure is based on not only the acoustic scores for the component sub-syllabic units of the keywords, but a set of word-length-dependent parameters trained with the minimum classification error criteria.
\end{abstract}

\section{INTRODUCTION}

In our previous research, by taking advantage of the monosyllabic structure of Mandarin Chinese, we have successfully developed a multi-phase approach for fast spotting of large vocabulary Chines keywords from Mandarin speech [1]. This approach includes four phases: The first phase is for detection of all possible syllable boundaries in the speech utterance, the second phase is for acoustic recognition of all possible syllable hypotheses and using these syllable hypotheses to construct a syllable lattice, the third phase is for keyword matching within the syllable lattice, while the fourth phase for re-scoring the keywords and extracting the most possible ones. This is because in Mandarin Chinese every character is a morpheme with its own meaning, pronounced as a monosyllable, while a word is composed of one to several characters, and the total number of phonologically allowed syllables is limited. Several specially designed likelihood score normalization techniques were then further developed to improve the spotting performance [2]. With these improved normalization techniques, a spotting rate of $85.79 \%$ for the top 10 candidates was achieved for a task with 2611 Chines keywords.

From the previous experiences, it was found that most false alarms were caused by shorter keywords (i.e. keywords with less syllables or characters), while the false rejections occur much more frequently for longer keywords (i.e. keywords with more syllables or characters) as compared to shorter keywords. These situations are apparently caused by the fact that the keyword length (i.e. the number of characters or syllables in the keywords) can vary very significantly among the keywords to be spotted. First, very often a shorter keyword is matched to an utterance segment which is either a homonym of the keyword, or is phonetically similar to the keyword, i.e. the utterance segment may differ from the keyword by only one or two phonemes or a single tone. The former problem for homonym segments can't be solved by acoustic process alone and occurs much less frequently in any case as compared to the latter problem of phonetically similar utterances, therefore, here we will consider only the latter problem. On the other hand, the 
problem of phonetically similar utterances is much less serious for longer words compared to shorter words, but instead the false rejection of longer keywords very often occurs when one or several syllables in the longer keyword are underestimated in acoustic scores or even misrecognized and lost in the constructed syllable lattice. This is because only task independent and speaker independent subsyllabic models are adopted in our approach in order to handle the large vocabulary and provide the vocabulary flexibility, which are often not good enough for acoustic recognition. However, it was observed that even though a long keyword has relatively low score, the possibility for the keyword is usually very high as long as its syllable string can be found in searching through the syllable lattice, even when one or two sub-syllabic units are lost in the string. Based on these observations, a confidence measure $[3,4,5]$ is believed to be very helpful in verifying the reliability of the word candidates. In this paper, such a confidence measure is proposed depending on the acoustic recognition results considering the different lengths of the keywords with some word-length-dependent parameters carefully trained.

The rest of this paper is organized as follows. In Section 2, we will first introduce the idea of word-length-dependent confidence measure and then propose two different methods to implement this idea. In Section 3, some experimental results to show the performances of the proposed methods are presented. Finally, in Section 4, some conclusions are drawn.

\section{WORD-LENGTH-DEPENDETN CONFIDENCE MEASURE}

The problem of keyword spotting can be defined as:

$$
\left(W_{k}^{*}, b_{1}^{*}, b_{2}^{*}\right)=\underset{\substack{\left(W_{k} b_{1}, b_{2}\right) \\ 0 \leq b_{1} b_{2} \leq T}}{\arg \max } \Phi_{k}\left(b_{1}, b_{2}\right)
$$

where $\Phi_{k}\left(b_{1}, b_{2}\right)$ is the normalized loglikelihood score of the speech utterance segment on the time interval $\left(b_{1}, b_{2}\right)$, for a given keyword $W_{k}$. The utterance segment $\left(b_{1}^{*}, b_{2}^{*}\right)$ for a certain keyword $W_{k}^{*}$ with the highest score $\Phi_{k}\left(b_{1}, b_{2}\right)$ within the complete utterance $(0, T)$ will be picked up as the result. Here, each keyword candidate obtained after the third phase of keyword matching through the syllable lattice as mentioned above is assigned a confidence measure. Assuming the $p$-th possible candidate obtained in the third phase is the $k$-th keyword in the vocabulary set, and this keyword candidate contains $i$ sub-syllabic units, the confidence measure for this $p$-th candidate is thus defined as:

$$
C M_{p}=\Phi_{k}\left(b_{1}, b_{2}\right) \times\left[1+a_{i}\left(n-\overline{n_{i}}\right)\right]
$$

where $n$ is the number of the sub-syllabic units in this keyword with acoustic scores exceeding a given threshold, $\overline{n_{i}}$ is the expected value of this number $n$ for all the possible keyword candidates with exactly $i$ sub-syllabic units found in this keyword searching phase, and $a_{i}$ is a weighting factor for keywords with exactly $i$ sub-syllabic units. Since both $\Phi_{k}\left(b_{1}, b_{2}\right)$ and $a_{i}$ are always positive, $C M_{p}>\Phi_{k}\left(b_{1}, b_{2}\right)$ if $n>\overline{n_{i}}$ and $C M_{p}<\Phi_{k}\left(b_{1}, b_{2}\right)$ if $n<\overline{n_{i}}$. So the purpose here is that in case of longer keywords for which the false rejection is likely to occur this confidence measure can be increased more, while for shorter keywords for which the false alarm is likely to occur this confidence measure can be more or less suppressed. In the following, two methods are proposed to obtain parameters $\overline{n_{i}}$ 's and $a_{i}$ 's. 


\subsection{Method 1}

The first method simply assumes that $\overline{n_{i}}$ for keywords with exactly $i$ sub-syllabic units is linearly proportional to $i$. That is to say:

$$
\overline{n_{i}}=\alpha \times i
$$

where $\alpha$ is a constant value. Besides, another single constant value $a_{0}$ is assigned to all weighting factors $a_{i}$ 's in equation (2).

\subsection{Method 2}

In stead of just using constant parameters, another method was further designed to better estimate all parameters directly from the training speech database. First, $\bar{n}_{i}$ is got by taking the average value of the number $n$ for all the possible keyword candidates with exactly $i$ sub-syllabic units found in the keyword searching phase for the training speech database. And the training procedure for the parameters $a_{i}$ 's is described below.

For the $p$-th candidate, another measure $\overline{C M_{p}^{-}}$is also defined, which is the average of the confidence measures for all the other candidates in the vocabulary set, except the $p$-th candidate, and then a difference value can be thus drawn:

$$
C M_{p}^{D}=C M_{p}-\overline{C M_{p}^{\bar{p}}}
$$

The training procedure is therefor to adjust $\bar{n}_{i}$ and $a_{i}$ iteratively by maximizing $C M_{p}^{D}$ when the $p$-th keyword candidate is correctly recognized and minimizing $C M_{p}^{D}$ when the $p$ th keyword candidate is incorrectly recognized. Here a monotonically increasing and differentiable sigmoid function is used for the minimum classification error criterion [6]. The loss function is defined as:

$$
L(A, \bar{N})=\frac{1}{1+e^{-\gamma\left(M_{P}^{D}(A, \bar{N})\right.}}
$$

where $A=\left(a_{1}, a_{2}, \cdots, a_{l}\right), \bar{N}=\left(\overline{n_{1}}, \overline{n_{2}}, \cdots, \overline{n_{I}}\right)$ are the vectors for the parameters $a_{i}$ and $\bar{n}_{i}$, $i=1,2, \ldots, I, I$ is the largest number of subsyllabic units in the keywords, and $\gamma$ is a constant for controlling the smoothness of the sigmoid function. In this way, both $\bar{N}$ and $A$ will be adjusted iteratively with the gradient descent method. For example, with the partial derivative of $L(A, \bar{N})$ with respective to $a_{i}, a_{i}$ can be adjusted by $\varepsilon \nabla E\{L(A, \bar{N})\}$, where $\varepsilon$ is a positive small learning factor.

\section{EXPERIMENTAL RESULTS}

A task with 2611 keywords was tested here. The 2611 keywords are actually the titles (or abbreviations of the titles) of all banding/financing organizations in Taipei area. The acoustic models used in this paper are trained from a collection of speech database recorded by 150 speakers. And the sub-syllabic units adopted here are initial's and final's, where "initial" is the initial consonant of a syllable, and "final" is everything in the syllable after the "initial", including the vowel or diphthong part plus optional medial or nasal ending. Since a syllable contains exactly an initial and a final, the number of sub-syllabic units $i$ for a work is exactly two times the number of characters in the word and it's the upper bound of $n$.

Table 1 shows the experimental results of method 1 in terms of the spotting rate for the top 10 candidates according to manually assigned $\alpha$ 's and $a_{0}$ 's. We can see that the best result will be achieved when $\alpha=0.2$ and 
$a_{0}=0.65$, and a spotting rate of $87.14 \%$ is obtained.

\begin{tabular}{|c|c|c|c|c|}
\hline$a_{0}$ & 0.6 & 0.65 & 0.7 & 0.75 \\
\hline 0.1 & $85.83 \%$ & $86.43 \%$ & $87.03 \%$ & $86.52 \%$ \\
\hline 0.2 & $86.13 \%$ & $86.84 \%$ & $87.14 \%$ & $86.73 \%$ \\
\hline 0.3 & $86.05 \%$ & $86.52 \%$ & $86.87 \%$ & $86.68 \%$ \\
\hline
\end{tabular}

Table 1. The experimental results of method 1 in terms of spotting rate for the top 10 candidates according to $\alpha$ 's and $a_{0}$ 's.

Figure 1 shows the experimental results in terms of the receiver operating characteristic (ROC) curves. The results for the test of the approach [1,2] without using the confidence measure mentioned here are also shown for comparison. The results for method 1 was tested with $\alpha=0.2$ and $a_{0}{ }^{\prime}=0.7$. The results show that the spotting performance can be improved by using the confidence measure. And a spotting rate of $88.76 \%$ for the top 10 candidates was achieved by using method 2 .

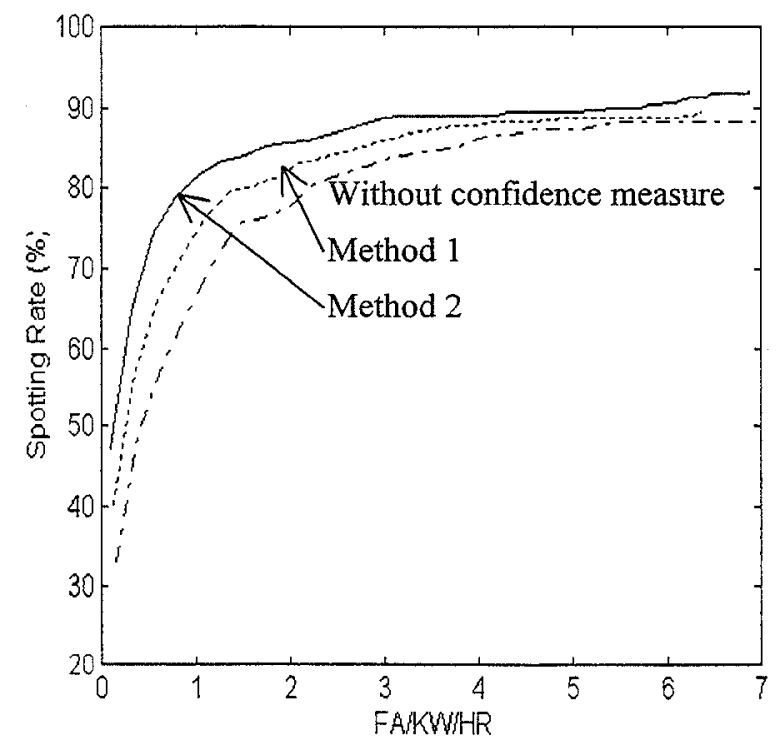

Figure 1. The experimental results for tests with and without confidence measure.

\section{CONCLUSION}

In this paper, we have proposed a wordlength-dependent confidence measure to improve the performance of large vocabulary Chinese keyword spotting. It can reduce the false alarms for shorter keywords and the false rejections for longer keywords. Besides acoustic scores and those word-length-dependent parameters used in this paper, we think that many other information such as duration of subsyllabic units, energy level, ...etc., are also useful for confidence measure. These will be considered in the further study.

\section{REFERENCES}

1. Bo-Ren Bai, Chiu-Yu Tseng, and Lin-Shan Lee, "A Multi-phase Approach for Fast Spotting of Large Vocabulary Chinese Keywords from Mandarin Speech Using Prosodic Information", Vol. 2, pp.903-906, ICASSP97.

2. Bo-Ren Bai, Hsin-Min Wang, and Lin-Shan Lee, "Log-likelihood Score Normalization Techniques for Recognition of Chinese Keywords with Large Vocabulary", Vol. 1, pp. 186-191, ICCPOL97.

3. Eduardo Lleida, and Richard C. Rose, "Likelihood Ratio Decoding and Confidence Measures for Continuous Speech Recognition", Vol. 1, pp. 478-481, ICSLP96.

4. Ze'ev Rivlin, Michael Cohen, Victor Abrach, and Thomas Chung, "A Phone-Dependent Confidence Measure for Utterance Rejection", Vol. 1, pp. 515-517, ICASSP96.

5. Thomas Schaaf and Thomas Kemp, "Confidence Measures for Spontaneous Speech Recognition", Vol. 2, pp. 875-878, ICASSP97.

6. Mazin G. Rahim, Chin-Hui Lee, BiingHwang Juang, and Wu Chou, "Discriminative Utterance Verification Using Minimum String Verification Error (MSVE) Training", Vol. 6, pp. 3585-3588, ICASSP96. 